\title{
Efeito do sinergista butóxido de piperonila na resistência de Oryzaephilus surinamensis (L.) (Coleoptera, Silvanidae) a deltametrina e fenitrotiom ${ }^{1}$
}

\author{
Helenara dos Santos Beckel², Irineu Lorini ${ }^{3}$ \& Sonia M. N. Lazzari ${ }^{4}$
}

${ }^{1}$ Contribuição $N^{\circ} 1557$ do Departamento de Zoologia, da Universidade Federal do Paraná. Parte da tese de Doutorado em Entomologia do $1^{\circ}$ autor, Convênio Embrapa Trigo-UFPR.

${ }^{2}$ R. Cel. Miranda, 651/603, 99025-050 Passo Fundo-RS. helenara@via-rs.net

${ }^{3}$ Embrapa Trigo-Centro Nacional de Pesquisa de Trigo; Caixa Postal 451, 99001-970 Passo Fundo-RS. ilorini@cnpt.embrapa.br

${ }^{4}$ Departamento de Zoologia, Universidade Federal do Paraná. Caixa Postal 19020, 81531-980 Curitiba-PR. lazzari@ufpr.br

\begin{abstract}
Synergistic effect of piperonyl butoxide on the resistance of Oryzaephilus surinamensis (L.) (Coleoptera, Silvanidae) to deltamethrin and fenitrothion. The use of synergists is an important tool to determine resistance mechanisms in insect species. In this research, the synergist piperonyl butoxide (PBO) was used, in different proportions, to evaluate the relative contribution of oxidase enzymes in the metabolism of the organophosphorous insecticide fenitrothion and the pyrethroid deltamethrin, on four Oryzaephilus surinamensis strains: OS1 (susceptible) and OS2, OS3 and OS4 (resistants). The synergist increased deltamethrin toxicity significantly in resistant strains, indicating that oxidases play an important role in deltamethrin resistance. On the other hand, PBO showed antagonistic effect to fenitrothion, reducing its toxicity significantly, indicating that this synergist is not adequate for mixtures with organophosphorous compounds.
\end{abstract}

KEYWORDS. Organophosphorous insecticide; pyrethroid insecticide; synergism.

RESUMO. Efeito do sinergista butóxido de piperonila na resistência de Oryzaephilus surinamensis (L.) (Coleoptera, Silvanidae) a deltametrina e fenitrotiom. A utilização de sinergistas é uma importante ferramenta para determinar os mecanismos envolvidos na resistência de insetos. Nesta pesquisa, o sinergista butóxido de piperonila (PBO) foi usado, em diferentes proporções, para avaliar a contribuição relativa de enzimas oxidases no metabolismo do inseticida organofosforado fenitrotiom e do piretróide deltametrina, em quatro populações de Oryzaephilus surinamensis: OS1 (suscetível) e OS2, OS3 e OS4 (resistentes). O sinergista aumentou, significativamente, a toxicidade da deltametrina nas populações resistentes, indicando que as oxidases exercem uma importante função na resistência a este inseticida. Para o fenitrotiom, o PBO apresentou um efeito antagonista, diminuindo significativamente a toxicidade do inseticida em todas as populações, indicando que este sinergista não é o mais apropriado para a mistura com compostos organofosforados.

PALAVRAS-CHAVE. Inseticidas organofosforados; inseticidas piretróides; sinergismo.

No Brasil, a espécie Oryzaephilus surinamensis (Linnaeus, 1758) (Coleoptera, Silvanidae) é encontrada praticamente em todas as unidades armazenadoras, onde por seus danos contribui para a deterioração de grãos (Lorini 2001).

Testes desenvolvidos com populações da praga, procedentes da região sul do Brasil (Beckel et al. 2002), detectaram níveis de resistência a inseticidas organofosforados e piretróides, amplamente utilizados para o controle das pragas de produtos armazenados. Entretanto, esses testes não determinam como se deve manejar as populações de pragas resistentes e, para desenvolver novas estratégias de controle da praga, é imprescindível entender melhor a origem e o desenvolvimento da resistência.

Em geral, os mecanismos de resistência de insetos a inseticidas são incluídos em três categorias: a) redução da penetração do inseticida pela cutícula do inseto; b) redução da sensibilidade no sítio de ação do inseticida pelo sistema nervoso; e c) detoxificação ou metabolização do inseticida por enzimas (Oppenoorth 1985; Hemingway 2000; Lorini \& Beckel 2002). O comportamento dos insetos frente a inseticidas é um quarto mecanismo que recentemente vem sendo estudado, e tem sido verificado pela repelência de inseticidas exercida sobre as pragas (Hodges \& Meik 1986), e por alterações de comportamento provocadas pelos químicos (Lorini \& Galley 1998; Beckel et al. 2004).

O metabolismo ou detoxificação é, provavelmente, o mecanismo mais estudado de resistência de insetos a inseticidas. Este mecanismo permite ao inseto modificar ou detoxificar o inseticida a uma taxa suficiente o bastante para prevenir a ação no sítio alvo (Fukuto \& Mallipudi 1983). A degradação do inseticida pode ocorrer por vários processos metabólicos nos quais o produto é convertido em uma forma não tóxica ou mesmo eliminado rapidamente do corpo do inseto. Várias enzimas e sistemas enzimáticos estão envolvidos, como as esterases, oxidases, transferases e outras enzimas que aumentam sua eficiência ou sua quantidade nas populações resistentes (Yu \& Nguyen 1992; Hemingway 2000). As oxidases e transferases são enzimas ubíquas que estão comumente envolvidas na detoxificação de numerosos compostos. Já as esterases são de maior importância, especificamente na detoxificação de organofosforados (Conyers et al. 1998). A resistência associada com esses processos é controlada primariamente por genes localizados no cromossomo II, na mosca doméstica, e parece ser herdada de uma maneira 
intermediária a parcialmente dominante (Plapp \& Wang 1983).

A interação entre mecanismos de detoxificação metabólica e espécies de insetos resistentes a inseticidas pode ser constatada através do uso de sinergistas (Wilkinson 1983; Hinks \& Spurr 1991), os quais representam uma importante ferramenta de laboratório (Raffa \& Priester 1985).

A ação do sinergista minimiza a quantidade de inseticida químico necessária para o controle de insetos, pois age como um substrato alternativo, poupando o inseticida da detoxificação, ou reage com outro sítio no sistema enzimático, prevenindo a detoxificação do inseticida (Casida 1970), aumentando assim a letalidade dos mesmos nas populações resistentes (Brindley \& Selim 1984). Além disso, os sinergistas, quando misturados com inseticidas, podem minimizar a contaminação ambiental dos resíduos de inseticidas persistentes e preservar insetos benéficos, como indicaram Raffa \& Priester (1985).

Os sinergistas têm sido intensamente empregados na tentativa de superar o problema de resistência e ajudar a controlar pragas no campo e, particularmente, em ambientes de grãos armazenados. Em algumas espécies de coleópteros de produtos armazenados houve comprovação da resistência bioquímica, como indicado por Subramanyam et al. (1989) utilizando inseticidas combinados com sinergistas. Esses autores observaram que a enzima carboxilesterase estava envolvida na detoxificação de malatiom em adultos resistentes de Tribolium castaneum (Herbst, 1797) (Coleoptera, Tenebrionidae); enquanto que oxidases e esterases são responsáveis pela detoxificação de clorpirifós-metil em adultos de $O$. surinamensis.

Lorini \& Galley (2000) mostraram que o sinergista butóxido de piperonila (PBO) aumentou a toxicidade do inseticida deltametrina em uma maneira dose-dependente, em todas as proporções testadas em populações resistentes de Rhyzopertha dominica (Fabricius, 1792) (Coleoptera, Bostrichidae), indicando que enzimas oxidases multifuncionais (MFO) são importantes na detoxificação desse inseticida. Entretanto, para populações suscetíveis, a adição de PBO aumentou a toxicidade somente nas maiores proporções testadas. Esses autores também testaram o sinergista DEF (S,S,S-tributilfosforotritioato) com deltametrina, nas mesmas populações de $R$. dominica, e não encontraram um efeito sinergista significativo, constatando que enzimas esterases possivelmente não estejam envolvidas na resistência a deltametrina em $R$. dominica.

Guedes et al. (1997a) demonstraram que a atividade específica da acetilcolinesterase diferiu significativamente entre populações resistentes e suscetíveis de $R$. dominica, indicando que a atividade aumentada da acetilcolinesterase parece estar associada com a resistência a organofosforados. E, com relação à resistência bioquímica aos piretróides, estudos de Collins (1990), com a espécie T. castaneum, indicam que o aumento do metabolismo enzimático na molécula do inseticida envolve oxidação e/ou hidrólise por ésteres.

Assim, informações sobre a toxicologia de inseticidas químicos e a identificação de mecanismos de resistência, no sentido de distinguir um sistema enzimático de outro, podem ser obtidas por investigações apropriadas com sinergistas. $\mathrm{O}$ butóxido de piperonila, o qual inibe as MFOs, tem sido usado como sinergista com inseticidas organofosforados e piretróides para controlar pragas de grãos armazenados com excelentes resultados (Samson et al. 1990; Daglish et al. 1995; Lorini \& Galley 2000).

A proposta deste estudo foi investigar os mecanismos de resistência em populações de $O$. surinamensis ao inseticida organofosforado fenitrotiom e ao piretróide deltametrina, com base em bioensaios utilizando o sinergista butóxido de piperonila.

\section{MATERIAL E MÉTODOS}

Quatro populações de $O$. surinamensis foram utilizadas nos experimentos: OS1, OS2 OS3 e OS4, coletadas em 1998, nos municípios de Passo Fundo-RS, Ubiratã-PR, Santo Ângelo-RS e Saldanha Marinho-RS, respectivamente.

A população OS1 foi considerada suscetível, e as populações OS2, OS3 e OS4, resistentes aos inseticidas fenitrotiom e deltametrina (Beckel et al. 2002).

Foram utilizados indivíduos da décima geração $\left(\mathrm{F}_{\mathrm{n}+10}\right)$ das populações OS1, OS2 e OS3, mantidos em grãos de trigo. Para a população OS4, indivíduos da nona geração $\left(\mathrm{F}_{\mathrm{n}+9}\right)$ foram utilizados para os testes com fenitrotiom, e da oitava geração $\left(\mathrm{F}_{\mathrm{n}+8}\right)$ para os testes com deltametrina.

Dois experimentos foram realizados, um com o inseticida fenitrotiom e outro com o inseticida deltametrina. Cada população foi testada sobre papel filtro impregnado com cinco concentrações de fenitrotiom (Sumigran $500 \mathrm{CE}$ ) e cinco de deltametrina (Decis $25 \mathrm{CE}$ ). As concentrações, de cada inseticida separadamente, foram combinadas com cinco proporções de PBO (1:0, 1:5, 1:10, 1:15 e 1:20), as quais foram calculadas sobre a quantidade de ingrediente ativo dos inseticidas utilizados em cada concentração. Ou seja, no primeiro experimento foi usada uma parte de fenitrotiom + zero de $\mathrm{PBO}$; uma parte de fenitrotiom +5 partes de $\mathrm{PBO}$; uma parte de fenitrotiom +10 partes de $\mathrm{PBO}$; uma parte de fenitrotiom + 15 partes de $\mathrm{PBO}$ e uma parte de fenitrotiom +20 partes de PBO. Para o segundo experimento foram usadas as mesmas proporções de deltametrina e $\mathrm{PBO}$ do primeiro experimento.

Os bioensaios foram executados seguindo o método recomendado pela FAO (1974), com algumas modificações para a espécie em estudo, em sala climatizada mantida a $25 \pm 0,5^{\circ} \mathrm{C}$ e $65 \pm 5 \%$ de UR. Cada população foi submetida a cinco tratamentos com o inseticida e um controle sem inseticida. Os inseticidas fenitrotiom $500 \mathrm{~g}$ i.a./L (Sumigran $500 \mathrm{CE}$ ) e deltametrina $25 \mathrm{~g}$ i.a./L (Decis $25 \mathrm{CE}$ ) foram diluídos em éter de petróleo, adicionado o sinergista PBO, para as concentrações estabelecidas ( $1 \%, 0,5 \%, 0,25 \%, 0,125 \%$ e $0,0625 \%$ ) e $1,0 \mathrm{ml} \mathrm{da}$ concentração foi distribuído sobre o papel filtro de $9 \mathrm{~cm}$ de diâmetro, colocado em placas de Petri, em quatro repetições. Após a evaporação do solvente, 10 insetos adultos, não sexados, de 1-20 dias de idade, foram liberados no interior de cada placa. 
Tabela I. Parâmetros de mortalidade para adultos de Oryzaephilus surinamensis das populações OS1, OS2, OS3 ( $\left.\mathrm{F}_{\mathrm{n}+10}\right)$ e OS4 ( $\left.\mathrm{F}_{\mathrm{n}+9}\right)$, testadas com fenitrotiom $(\mathrm{Fe})(1 \%, 0,5 \%, 0,25 \%, 0,125 \%$ e $0,0625 \%)+$ diferentes proporções de butóxido de piperonila $(\mathrm{PBO})$, a $25 \pm 0,5^{\circ} \mathrm{C}$ e UR de $65 \pm 5 \%$. Valores da $\mathrm{CL}_{50}$ em $\mathrm{mg} / \mathrm{cm}^{2}$ de fenitrotiom. Passo Fundo, RS, 2003.

\begin{tabular}{|c|c|c|c|c|c|c|}
\hline Proporcão Fe + PBO & $\mathrm{CL}_{50}(95 \% \lim . \text { conf. })^{1}$ & $\mathrm{a}$ & $\mathrm{EP}_{\mathrm{a}}$ & $\mathrm{b}$ & $\mathrm{EP}_{\mathrm{b}}$ & Razão $^{2}$ \\
\hline \multicolumn{7}{|l|}{ OS1: } \\
\hline $1+00$ & $0,4653(0,036-1,017) \mathrm{A}$ & 0,5063 & 0,2664 & 1,524 & 0,4464 & - \\
\hline $1+05$ & $0,2724(0,000-0,842) \mathrm{A}$ & 0,6654 & 0,2696 & 1,178 & 0,4295 & 1,7 \\
\hline $1+10$ & $9,8650(1,673-18,49) \mathrm{B}$ & $-1,2990$ & 0,5951 & 1,307 & 0,3794 & $-21,2$ \\
\hline $1+15$ & $15,650(0,000-38,66) \mathrm{C}$ & $-0,8613$ & 0,5585 & 0,721 & 0,3423 & $-33,6$ \\
\hline $1+20$ & $19,640(11,86-27,74) \mathrm{C}$ & $-2,7170$ & 0,6276 & 2,101 & 0,4043 & $-42,2$ \\
\hline \multicolumn{7}{|l|}{ OS2: } \\
\hline $1+00$ & $1,556(1,128-1,979) \mathrm{B}$ & $-0,6873$ & 0,2761 & 3,578 & 0,5680 & - \\
\hline $1+05$ & $1,168(0,218-2,147) \mathrm{A}$ & $-0,0845$ & 0,2481 & 1,255 & 0,3703 & 1,3 \\
\hline $1+10$ & $20,29(7,365-34,24) \mathrm{C}$ & $-1,6780$ & 0,5776 & 1,284 & 0,3588 & $-13,0$ \\
\hline $1+15$ & $37,55(16,29-81,68) \mathrm{C}$ & $-1,6680$ & 0,5706 & 1,059 & 0,3466 & $-24,1$ \\
\hline $1+20$ & $87,87(47,71-422,8) \mathrm{D}$ & $-2,1330$ & 0,5949 & 1,097 & 0,3536 & $-56,4$ \\
\hline \multicolumn{7}{|c|}{ - } \\
\hline $1+00$ & $2,721(2,028-3,522) \mathrm{A}$ & $-1,276$ & 0,2855 & 2,936 & 0,4501 & - \\
\hline $1+05$ & $8,197(4,856-23,73) \mathrm{B}$ & $-1,182$ & 0,2742 & 1,294 & 0,3606 & $-3,0$ \\
\hline $1+10$ & $66,93(44,53-123,8) \mathrm{C}$ & $-2,102$ & 0,4164 & 1,151 & 0,2489 & $-245,9$ \\
\hline $1+15$ & $226,4(118,3-1148,0) \mathrm{C}$ & $-2,524$ & 0,4544 & 1,072 & 0,2648 & $-832,0$ \\
\hline $1+20$ & $192,9(101,0-1044,0) \mathrm{C}$ & $-2,248$ & 0,4377 & 0,983 & 0,2572 & $-708,9$ \\
\hline \multicolumn{7}{|c|}{ (1) } \\
\hline $1+00$ & $2,265(1,545-3,050) \mathrm{A}$ & $-0,875$ & 0,2667 & 2,464 & 0,4230 & - \\
\hline $1+05$ & $6,395(4,813-9,076) \mathrm{B}$ & $-2,091$ & 0,3362 & 2,595 & 0,4295 & $-2,8$ \\
\hline $1+10$ & $84,72(61,91-133,2) \mathrm{C}$ & $-3,161$ & 0,4583 & 1,640 & 0,2677 & $-37,4$ \\
\hline $1+15$ & $129,9(88,97-245,9) \mathrm{C}$ & $-3,343$ & 0,4819 & 1,581 & 0,2770 & $-57,3$ \\
\hline $1+20$ & $74,65(58,65-101,3) \mathrm{C}$ & $-4,032$ & 0,4957 & 2,153 & 0,2867 & $-32,9$ \\
\hline
\end{tabular}

${ }^{1} \mathrm{CL}_{50}$ seguidas da mesma letra não são significativamente diferentes $(\mathrm{P}>0.05)$ dentro da mesma população. As séries logit log-dose foram comparadas pelo teste $\mathrm{F}$.

${ }^{2}$ Razão $=\mathrm{CL}_{50}$ sem PBO dividida pela $\mathrm{CL}_{50}$ com PBO, dentro de cada população. $\mathrm{a}=$ interseção $\mathrm{b}=$ inclinação $\mathrm{EP}=$ Erro Padrão

A avaliação da mortalidade, visando estabelecer as concentrações letais $\left(\mathrm{CL}_{50}\right)$ para cada população, foi feita pela contagem do número de insetos vivos e mortos 24 h após o tratamento, considerando-se mortos os insetos que não puderam caminhar normalmente durante um período de observação de 2 minutos. Para determinação da $\mathrm{CL}_{50}$ para cada população, os resultados de mortalidade dos bioensaios foram analisados pelo programa estatístico GLIM, Royal Statistical Society, versão 3.77 (Crawley 1993). A razão entre as CLs ${ }_{50}$ das diferentes proporções do sinergista e o inseticida, foram calculadas.

\section{RESULTADOS E DISCUSSÃO}

\section{Fenitrotiom + Butóxido de Piperonila}

$\mathrm{O}$ PBO adicionado ao inseticida fenitrotiom aumentou a dose letal média nas populações suscetível e resistentes, diminuindo muito a toxicidade do inseticida e demonstrando a ausência do efeito sinergista (Tabela I). As razões entre $\mathrm{CL}_{50}$ sem PBO e a $\mathrm{CL}_{50}$ com o PBO indicaram um efeito antagonista, resultando na eliminação dos efeitos nocivos do inseticida.

Os compostos organofosforados são conhecidamente metabolizados por enzimas esterases (Guedes et al. 1997b). Estudos de Conyers et al. (1998) demonstraram uma superprodução de esterase em uma população de $O$. surinamensis resistente aos organofosforados malatiom e fenitrotiom. Pode-se inferir que a ausência do efeito sinergista do PBO indica que o mesmo não é um inibidor efetivo de esterases nesta espécie. Entretanto, em estudos de Attia \& Frecker (1984), com populações de $O$. surinamensis resistentes a inseticidas organofosforados, o sinergista PBO sinergizou os inseticidas malatiom, fenitrotiom, clorpirifós-metil e pirimifósmetil testados.

Estudos de Guedes \& Zhu (1998) demonstraram que o PBO apresentou um efeito antagonista ao inseticida organofosforado malatiom em populações resistentes e suscetíveis de $R$. dominica. Segundo os autores, o malatiom precisa ser ativado pelo citocromo $\mathrm{P} 450$ para produzir malaoxon e então inibir a acetilcolinesterase. No exemplo mencionado, o efeito antagonista do PBO ao malatiom inibiu a ativação do sistema.

É importante salientar que para controlar pragas de grãos armazenados, misturas de inseticidas piretróides, que contêm o sinergista $\mathrm{PBO}$, com inseticidas organofosforados são utilizadas freqüentemente. Considerando-se os resultados encontrados neste trabalho, que indicam que PBO exerce um efeito antagonista quando associado ao organofosforado fenitrotiom, pode-se esperar que estas misturas apresentem eficiência reduzida no controle de $O$. surinamensis.

Segundo Wilkinson (1983), a avaliação do papel de oxidação no metabolismo de compostos organofosforados é difícil pelo fato de que em muitos casos, os produtos gerados pela ação 
Tabela II. Parâmetros de mortalidade para adultos de Oryzaephilus surinamensis das populações OS1, OS2, OS3 ( $\left.\mathrm{F}_{\mathrm{n}+10}\right)$ e OS4 ( $\left.\mathrm{F}_{\mathrm{n}+8}\right)$, testadas com deltametrina (Del) $(1 \%, 0,5 \%, 0,25 \%, 0,125 \%$ e $0,0625 \%)+$ diferentes proporções de butóxido de piperonila (PBO), a $25 \pm 0,5^{\circ} \mathrm{C}$ e UR de $65 \pm$ 5\%. Valores da $\mathrm{CL}_{50} \mathrm{em} \mathrm{mg} / \mathrm{cm}^{2}$ de deltametrina. Passo Fundo, RS, 2003.

\begin{tabular}{clccccc}
\hline Proporcão Del + PBO & $\mathrm{CL}_{50}(95 \% \text { lim. conf. })^{1}$ & $\mathrm{a}$ & $\mathrm{EP}_{\mathrm{a}}$ & $\mathrm{b}$ & $\mathrm{EP}_{\mathrm{b}}$ & Razão $^{2}$ \\
\hline OS1: & & & & & & \\
$1+00$ & $3,344(2,258-4,771) \mathrm{BC}$ & $-1,0590$ & 0,2692 & 2,019 & 0,3851 & - \\
$1+05$ & $1,723(1,003-2,447) \mathrm{AB}$ & $-0,5048$ & 0,2567 & 2,136 & 0,4136 & 1,9 \\
$1+10$ & $4,722(2,795-8,921) \mathrm{C}$ & $-0,9164$ & 0,2615 & 1,359 & 0,3563 & 0,7 \\
$1+15$ & $0,611(0,024-1,389) \mathrm{A}$ & 0,2503 & 0,2531 & 1,172 & 0,3872 & 5,4 \\
$1+20$ & $2,232(1,536-2,987) \mathrm{ABC}$ & $-0,8844$ & 0,2676 & 2,536 & 0,4290 & 1,5 \\
OS2: & & & & & & \\
$1+00$ & $19,55(13,950-25,41) \mathrm{E}$ & $-3,854$ & 0,7078 & 2,985 & 0,4757 & 1,7 \\
$1+05$ & $11,54(6,7610-15,95) \mathrm{D}$ & $-2,995$ & 0,7352 & 2,820 & 0,5218 & 8,4 \\
$1+10$ & $2,316(1,1840-3,585) \mathrm{C}$ & $-0,567$ & 0,2526 & 1,556 & 0,3683 & 21,5 \\
$1+15$ & $0,906(0,1037-1,794) \mathrm{B}$ & 0,052 & 0,2494 & 1,221 & 0,3762 & - \\
$1+20$ & $0,456(0,0067-1,160) \mathrm{A}$ & 0,392 & 0,2573 & 1,150 & 0,3977 & 42,8 \\
OS3: & & & & & & \\
$1+00$ & $61,19(45,96-86,58) \mathrm{D}$ & $-4,580$ & 0,7306 & 2,564 & 0,4253 & - \\
$1+05$ & $24,36(18,88-30,49) \mathrm{C}$ & $-4,978$ & 0,7746 & 3,590 & 0,5147 & 2,5 \\
$1+10$ & $8,026(6,345-10,53) \mathrm{AB}$ & $-3,039$ & 0,4390 & 3,360 & 0,4937 & 5,6 \\
$1+15$ & $10,33(7,835-14,93) \mathrm{B}$ & $-2,858$ & 0,4261 & 2,819 & 0,4644 & 9,4 \\
$1+20$ & $6,458(4,048-11,78) \mathrm{A}$ & $-1,201$ & 0,2973 & 1,482 & 0,3615 & - \\
OS4: & & & & & & \\
$1+00$ & $47,58(36,89-62,41) \mathrm{C}$ & $-5,062$ & 0,7569 & 3,018 & 0,4507 & \\
$1+05$ & $15,71(9,828-21,49) \mathrm{B}$ & $-3,035$ & 0,6737 & 2,537 & 0,4538 & 3,0 \\
$1+10$ & $3,829(3,065-4,729) \mathrm{A}$ & $-2,297$ & 0,3765 & 3,939 & 0,5353 & 12,4 \\
$1+15$ & $3,360(2,367-4,509) \mathrm{A}$ & $-1,301$ & 0,3061 & 2,471 & 0,4165 & 14,1 \\
$1+20$ & $3,438(2,427-4,619) \mathrm{A}$ & $-1,320$ & 0,3066 & 2,460 & 0,4150 & 13,8 \\
\hline
\end{tabular}

${ }^{1} \mathrm{CLs}_{50}$ seguidas da mesma letra não são significativamente diferentes $(\mathrm{P}>0.05)$ dentro da mesma população. As séries logit log-dose foram comparadas pelo teste $\mathrm{F}$.

${ }^{2}$ Razão $=\mathrm{CL}_{50}$ sem PBO dividida pela $\mathrm{CL}_{50}$ com $\mathrm{PBO}$, dentro de cada população. $\mathrm{a}=$ interseção $\mathrm{b}=$ inclinação $\mathrm{EP}=$ Erro Padrão

de oxidases são os mesmos resultantes da ação de outros tipos de enzimas, como as esterases e transferases.

Jao \& Casida (1974) indicam que o sinergista DEF é o melhor de uma série de sinergistas para compostos organofosforados devido ao seu forte efeito inibitório sobre esterases. Assim, para aprofundar os estudos aqui apresentados, sugere-se investigar o potencial das enzimas esterases na detoxificação de inseticidas organofosforados em $O$. surinamensis.

\section{Deltametrina + Butóxido de Piperonila}

O PBO aumentou a toxicidade da deltametrina em todas as proporções nas populações resistentes. Entretanto, para a população suscetível OS1, a adição do sinergista não conferiu relação de toxicidade, pois na combinação 1:15 a razão entre a $\mathrm{CL}_{50}$ sem $\mathrm{PBO}$ e a $\mathrm{CL}_{50}$ com $\mathrm{PBO}$ foi de 5,4 vezes enquanto que na proporção 1:20 foi somente de 1,5 vezes (Tabela II). Este resultado era esperado, pois, presume-se que os indivíduos desta população tenham expressado sua tolerância normal ao inseticida. O PBO conferiu maiores relações de toxicidade nas populações resistentes, e a maior razão entre $\mathrm{CL}_{50} \mathrm{sem} \mathrm{PBO}$ e a $\mathrm{CL}_{50}$ com $\mathrm{PBO}$ foi encontrada na população OS2, onde a relação de sinergismo na proporção 1:20 foi de 42,8 vezes (Tabela II).

Resultado similar foi encontrado por Lorini \& Galley (2000), que testaram o sinergista $\mathrm{PBO}$ com deltametrina em populações suscetíveis e resistentes de $R$. dominica, não encontrando alteração de toxicidade para as populações suscetíveis, e demonstrando efeito sinergista do PBO nas populações resistentes.

A adição do PBO à deltametrina reduziu efetivamente a dose letal média das populações resistentes de $O$. surinamensis testadas. A proporção 1:20 de deltametrina e sinergista reduziu a razão entre as $\mathrm{CL}_{50}$ da população suscetível (OS1) e da resistente (OS3) de 18 para 2 vezes. Como a resistência não foi completamente suprimida, é possível inferir que outro mecanismo de resistência, além do metabolismo por oxidases, possa também estar envolvido com essas populações. Além disso, os indivíduos testados já poderiam ter desenvolvido resistência às combinações de $\mathrm{PBO}$ e deltametrina, já que o $\mathrm{PBO}$ vem sendo adicionado ao inseticida deltametrina na proporção 1:10 em formulações comerciais (K-OBIOL $25 \mathrm{CE}$ ). Esse tratamento é feito em grãos armazenados para reduzir a taxa de aplicação sobre populações suscetíveis e também como uma medida mais econômica, com a vantagem de reduzir os níveis de resíduos nos grãos.

Como o PBO sinergizou o inseticida deltametrina neste experimento e, já que o mesmo, conhecidamente, bloqueia oxidases multifuncionais (Casida 1970), conclui-se que essas enzimas exercem uma importante função na resistência à deltametrina nas populações de $O$. surinamensis avaliadas neste estudo.

$\mathrm{O}$ uso de $\mathrm{PBO}$ mostrou ser uma ferramenta importante no 
estudo da resistência das pragas aos inseticidas, pois permite inferir sobre o mecanismo da resistência envolvido com a espécie e inseticida em estudo. Outros estudos podem ser necessários para identificar os demais mecanismos da resistência envolvidos, que não somente o metabolismo por enzimas. No entanto, a despeito das limitações discutidas anteriormente, os sinergistas como PBO, podem ser valiosos nos programas de manejo integrado de pragas, tendo em vista que já existem formulações comerciais de inseticidas contendo uma proporção do sinergista, visando aumentar a eficácia do inseticida.

\section{REFERÊNCIAS}

Attia, F. I. \& T. Frecker 1984. Cross-resistance spectrum and synergism studies in organophosphorus-resistant strains of Oryzaephilus surinamensis (L.) (Coleoptera: Cucujidae) in Australia. Journal of Economic Entomology 77: 1367-1370.

Beckel, H.; I. Lorini \& S. M. N. Lazzari. 2002. Detecção da resistência de Oryzaephilus surinamensis (L.) (Coleoptera: Silvanidae), praga de grãos de cevada armazenada, a inseticidas químicos, p. 620-630. In: E.Minella (ed.) Anais da XXII Reunião Anual de Pesquisa de Cevada. Embrapa Trigo, Passo Fundo, RS e http:// www.cnpt.embrapa.br

Beckel, H.; I. Lorini \& S. M. N. Lazzari. 2004. Comportamento de adultos de diferentes raças de Rhyzopertha dominica (Fabricius) (Coleoptera, Bostrichidae) em superfície tratada com deltamethrin. Revista Brasileira de Entomologia 48: 115-118.

Brindley, W. A. \& A. A. Selim. 1984. Synergism and antagonism in the analysis of insecticide resistance. Environmental Entomology 13: $348-353$.

Casida, J. E. 1970. Mixed-function oxidase involvement in the biochemistry of insecticide synergists. Journal of Agricultural and Food Chemistry 18: 753-772.

Collins, P. J. 1990. A new resistance to pyrethroids in Tribolium castaneum (Herbst). Pesticide Science 28: 101-115.

Conyers, C. M.; A. D. Macnicoll \& N. R. Price. 1998. Purification and characterisation of an esterase involved in resistance to organophosphorus insecticides in the saw-toothed grain beetle, Oryzaephilus surinamensis (Coleoptera: Silvanidae). Insect Biochemistry and Molecular Biology 28: 435-448.

Crawley, M. J. 1993. Glim for ecologists. Oxford, Blackwell Scientific Publications, $379 \mathrm{p}$.

Daglish, G. J.; M. Eelkema \& L. M. Harrison. 1995. Chlorpyrifosmethyl plus either methoprene or synergized phenothrin for control of Coleoptera in maize in Queensland, Australia. Journal of Stored Products Research 31: 235-241.

FAO 1974. Recommended methods for the detection and measurement of resistance of agricultural pest to pesticides: Tentative method for adults of some major beetle pest of stored cereals with malathion or lindane FAO Method $\mathrm{N}^{\circ} 15$. FAO Plant Protection Bulletin 22: $127-137$

Fukuto, T. R. \& N. M. Mallipudi. 1983. Supression of metabolic resistance through chemical structure modification, p. 557-578. In: G. P. Georghiou \& T. Saito (ed.). Pest resistance to pesticides: challenges and prospects. Plenum Press, New York, United States of America.

Guedes, R. N. C.; S. Kambhampati; B. A. Dover \& K. Y. Zhu. 1997a. Biochemical mechanisms of organophosphate resistance in Rhyzopertha dominica (Coleoptera: Bostrichidae) populations from the United State and Brazil. Bulletin of Entomological Research 87: $581-586$
Guedes, R. N. C. \& K. Y. Zhu.1998. Characterization of malathion resistance in a mexican population of Rhyzopertha dominica. Pesticide Science 53: 15-20.

Guedes, R. N. C.; K. Y. Zhu; B. A. Dover \& S. Kambhampati. 1997 . Partial characterization of phosphotriesterases from organophosphate-susceptible and resistant populations of Rhyzopertha dominica (Coleoptera: Bostrichidae). Pesticide Biochemistry and Physiology 57: 156-164.

Hemingway, J. 2000. The molecular basis of two contrasting metabolic mechanisms of insecticide resistance. Insect Biochemistry and Molecular Biology 30: 1009-1015.

Hinks, C. F. \& D. T. Spurr. 1991. The efficacy and cost benefits of binary mixtures of deltamethrin combined with other insecticides or synergists against grasshopers at two temperatures. Journal of Agricultural Entomology 8: 29-39.

Hodges, R. J. \& J. Meik. 1986. Lethal and sublethal effects of permethrin on Tanzanian strains of Tribolium castaneum (Herbst), Gnatocerus maxillosus (F.) Sitophilus oryzae (L.) and Sitophilus zeamais Motschulsky. Insect Science and Its Application 7: $533-537$.

Jao, L. T. \& J. E. Casida. 1974. Esterase inhibitors as synergists for (+)trans-chrysanthemate insecticide chemicals. Pesticide Biochemistry and Physiology 4: 456-464.

Lorini, I. 2001. Manual Técnico para o Manejo Integrado de Pragas de Grãos de Cereais Armazenados. Embrapa Trigo. Passo Fundo, RS. 80 p.

Lorini, I. \& H. S. Beckel. 2002. Mecanismos de resistência das pragas de grãos armazenados, p. 555-568. In: Lorini, L. H. Miike \& V. M. Scussel, Bio Geneziz, (ed.) Armazenagem de Grãos, Campinas, SP. 983 p.

Lorini, I. \& D. J. Galley. 1998. Relative effectiveness of topical, filter paper and grain applications of deltamethrin, and associated behaviour of Rhyzopertha dominica (F.) strains. Journal of Stored Products Research 34: 377-383.

Lorini, I. \& D. J. Galley. 2000. Effect of the synergists piperonyl butoxide and DEF in deltamethrin resistance on strains of Rhyzopertha dominica (F.) (Coleoptera: Bostrychidae). Anais da Sociedade Entomológica do Brasil 29: 749-755.

Oppenoorth, F. J. 1985. Biochemistry and genetics of insecticide resistance. Comprehensive insect physiology, biochemistry and pharmacology, p. 731-773. In: G. A. Kerkut \& L. I. Gilbert (ed.). Pergamon Press, Oxford, United Kingdom.

Plapp, F. W. \& T. C. Wang. 1983. Genetic origins of insecticide resistance, p. 47-70. In: G. P. Georghiou \& T. Saito (ed.). Pest resistance to pesticides: challenges and prospects. Plenum Press, New York, United States of America.

Raffa, K. F. \& T. M. Priester. 1985. Synergists as research tools and control agents in agriculture. Journal of Agricultural Entomology 2: 27-45.

Samson, P. R.; R. J. Parker \& E. A. Hall 1990. Synergized deltamethrin as a protectant against Sitophilus zeamais Motsch. and S. oryzae (L.) (Coleoptera: Curculionidae) on stored maize. Journal of Stored Products Research 26: 155-161.

Subramanyam, B.; P. K. Harein \& L. K. Cutkomp. 1989. Organophosphate resistance in adults of red flour beetle (Coleoptera: Tenebrionidae) and sawtoothed grain beetle (Coleoptera: Cucujidae) infesting barley stored on farms in Minnesota. Journal of Economic Entomology 82: 989-995.

Wilkinson, C. F. 1983. Role of mixed-function oxidases in insecticide resistance, p. 175-205. In: G. P. Georgiou \& T. Saito (ed.). Pest Resistance to Pesticides: Challenges and Prospects. Plenum Press, New York, United States of America.

Yu, S. J. \& S. N. Nguyen. 1992. Detection and biochemical characterization of insecticide resistance in the diamondback moth Pesticide Biochemistry and Physiology 44: 74-81.

Recebido em 18/03/2005; aceito em 09/01/2006 